\title{
Aromatase Controls Sjögren Syndrome-Like Lesions through Monocyte Chemotactic Protein-1 in Target Organ and Adipose Tissue-Associated Macrophages
}

\author{
Akihiko Iwasa, ${ }^{\dagger \dagger}$ Rieko Arakaki, ${ }^{*}$ Naoko Honma,${ }^{\ddagger}$ Aya Ushio, ${ }^{*}$ Akiko Yamada, ${ }^{*}$ Tomoyuki Kondo, ${ }^{*}$ Emi Kurosawa, ${ }^{*}$ \\ Satoko Kujiraoka, ${ }^{*}$ Takaaki Tsunematsu, ${ }^{*}$ Yasusei Kudo, ${ }^{*}$ Eiji Tanaka, ${ }^{\dagger}$ Noriko Yoshimura, ${ }^{\S}$ Nobuhiro Harada, ${ }^{\S}$ Yoshio Hayashi, ${ }^{*}$ \\ and Naozumi Ishimaru*
}

\begin{abstract}
From the Departments of Oral Molecular Pathology* and Orthodontics and Dentofacial Orthopedics, ${ }^{\dagger}$ Institute of Health Biosciences, The University of Tokushima Graduate School, Tokushima; the Research Team for Geriatric Pathology, ${ }^{\ddagger}$ Tokyo Metropolitan Institute of Gerontology, Tokyo; and the Department of Biochemistry, ${ }^{\S}$ School of Medicine, Fujita Health University, Toyoake, Japan
\end{abstract}

\author{
Accepted for publication \\ September 9, 2014. \\ Address correspondence to \\ Naozumi Ishimaru, D.D.S. \\ Ph.D., Department of Oral \\ Molecular Pathology, Institute \\ of Health Biosciences, The \\ University of Tokushima Grad- \\ uate School, 3-18-15 \\ Kuramotocho, Tokushima \\ 770-8504, Japan. E-mail: \\ ishimaru.n@tokushima-u.ac.jp.
}

\begin{abstract}
Several autoimmune diseases are known to develop in postmenopausal women. However, the mechanism by which estrogen deficiency influences autoimmunity is unknown. Aromatase is an enzyme that converts androgens to estrogens. Herein, we used female aromatase gene knockout (ArKO) mice as a model of estrogen deficiency to investigate the molecular mechanism that underlies the onset and development of autoimmunity. Histological analyses showed that inflammatory lesions in the lacrimal and salivary glands of ArKO mice increased with age. Adoptive transfer of spleen cells or bone marrow cells from ArKO mice into recombination activating gene 2 knockout mice failed to induce the autoimmune lesions. Expression of mRNA encoding proinflammatory cytokines and monocyte chemotactic protein-1 increased in white adipose tissue of ArKO mice and was significantly higher than that in wildtype mice. Moreover, an increased number of inflammatory M1 macrophages was observed in white adipose tissue of ArKO mice. A significantly increased monocyte chemotactic protein-1 mRNA expression of the salivary gland tissue in ArKO was found together with adiposity. Furthermore, the autoimmune lesions in a murine model of Sjögren syndrome were exacerbated by administration of an aromatase inhibitor. These results suggest that aromatase may play a key role in the pathogenesis of Sjögren syndrome-like lesions by controlling the target organ and adipose tissue-associated macrophage. (Am J Pathol 2015, 185: 151-161; http://dx.doi.org/10.1016/j.ajpath.2014.09.006)
\end{abstract}

Autoimmune disease is caused by multiple factors. One of them is the disruption of self-tolerance. ${ }^{1,2}$ Several autoimmune diseases, such as rheumatoid arthritis or Sjögren syndrome (SS), occur in postmenopausal women. Sex steroid hormones, such as estrogens, may influence the onset or development of autoimmune diseases through an unknown mechanism. ${ }^{3,4}$

SS is characterized by a 9:1 ratio of women/men, and almost all SS patients are postmenopausal women. ${ }^{5} \mathrm{SS}$ targets exocrine glands, such as the salivary and lacrimal glands, causing patients with SS to experience dryness of the mouth and eyes. ${ }^{6} \mathrm{We}$ demonstrated that estrogen deficiency caused by ovariectomy exacerbates autoimmune lesions in a murine model of SS. 7 Furthermore, we reported that retinoblastoma-associated protein 48 ( $\mathrm{RbAp} 48)$ induces tissue-specific apoptosis in the salivary and lacrimal glands of mice, depending on the level of estrogen deficiency. ${ }^{8}$ Moreover, we observed that transgenic expression of $R b A p 48$ in exocrine glands causes the development of autoimmune exocrinopathy, resembling SS. ${ }^{9}$ Although postmenopausal estrogen deficiency triggers the breakdown of immune tolerance and induces autoimmune disease, multiple direct or indirect factors

Supported, in part, by the Funding Program for Next Generation WorldLeading Researchers in Japan grant LS090, Ministry of Education, Science, Sport, and Culture of Japan Grants-in-Aid for Scientific Research 25670798, 24659839, and 23390429, and the Uehara Memorial Foundation.

Disclosures: None declared. 
that are affected by the change in estrogen level impede efforts to understand the molecular mechanisms involved in autoimmunity.

Estrogens are synthesized from androgens by aromatase, a key step in the biosynthesis of estrogens. Studies on aromatase gene knockout (ArKO) mice show that aromatase/estrogens play key roles in controlling sex-specific morphological, neuroendocrinological, and behavioral differences in the brain. ${ }^{10-12}$ Furthermore, ArKO mice develop marked abdominal adiposity, suggesting that aromatase/estrogens control the adipose phenotype through the regulation of lipid metabolism. ${ }^{13,14}$ Moreover, ArKO mice spontaneously develop an autoimmune disease, resembling age-associated SS lesions through B-cell hyperplasia and autoantibody production. ${ }^{15}$ In addition, increased numbers of peripheral blood cells, bone marrow cells, and inflammatory renal lesions are observed in ArKO mice. ${ }^{15}$ However, the molecular mechanism responsible for the onset of autoimmunity in exocrine glands through aromatase is unknown. In particular, the relationship between aromatase activity and the function of immune cells, in addition to B cells, in the onset or development of autoimmune disease must be determined to understand the dysfunction of the immune system in postmenopausal women.

Herein, we investigated the association of the pathogenesis of autoimmune disease with postmenopausal changes induced by estrogen deficiency. Furthermore, we attempted to define the molecular mechanism that accounts for the breakdown of peripheral immune tolerance with the goal of establishing a new therapeutic strategy to treat sex-specific autoimmune diseases.

\section{Materials and Methods}

Mice

Female C57BL/6 (B6) mice were purchased from the Japan SLC Laboratory (Shizuoka, Japan). ArKO mice were obtained from RIKEN BioResource Center (Tsukuba, Japan). Female $A r \mathrm{KO}$ were used for this study. Female recombination activating gene 2 knockout (Rag2KO) mice were obtained from Taconic (Germantown, NY). Female NFS/N mice carrying mutant sld were reared in our specific pathogen-free mouse colony and given food and water ad libitum. Thymectomy was performed on day 3 after birth of the NFS/sld mice. All mice were bred in the animal facility of the University of Tokushima (Tokushima, Japan) under specific pathogen-free conditions. The experiments were approved by the animal ethics board of the University of Tokushima.

\section{Administration of $\mathrm{AI}$}

The aromatase inhibitor (AI) exemestane (Sigma-Aldrich, St. Louis, MO) was prepared as an aqueous suspension in $0.5 \%$ carboxymethylcellulose (CMC; Dai-Ichi Chemical Industries, Tokyo, Japan). The i.p. injection of B6 mice
(8 weeks old) with $200 \mu \mathrm{L} \mathrm{CMC}$, containing AI (0, 50, and $200 \mu \mathrm{g}$ per mouse), was administered. For female thymectomized (Tx)-NFS/sld mice, AI in CMC was injected every day until 4 to 8 weeks of age.

\section{Histological Features}

All organs were removed from the mice, fixed with $4 \%$ phosphate-buffered formaldehyde $(\mathrm{pH} 7.2)$, and prepared for histological examination. Formalin-fixed tissue sections were subjected to hematoxylin and eosin (H\&E) staining, and three pathologists (N.I., Y.K., A.Y.) independently evaluated the histological features without being informed of the condition of each mouse. Histological grading was performed according to a previously published method. ${ }^{16}$ In brief, the inflammatory lesions of lacrimal and salivary glands were evaluated as followed: slight, no change or slight lymphoid cell infiltration; moderate, lymphoid cell infiltration; and severe, marked lymphoid cell infiltration with tissue destruction.

\section{Adoptive Cell Transfer}

Spleen cells $\left(5 \times 10^{6}\right)$ from female wild-type (WT) and ArKO mice (10 weeks of age) were i.v. transferred into female Rag2KO mice ( 8 weeks of age). In addition, bone marrow cells $\left(5 \times 10^{6}\right)$ from WT and ArKO mice were i.v. transferred into irradiated (8-Gy) Rag2KO mice. Twelve weeks after transfer, the pathological features of all organs were analyzed.

\section{Flow Cytometry}

Antibodies against CD4, CD8, CD11c, F4/80, CCR2, and CD206 were conjugated as required to fluorescein isothiocyanate, phosphatidylethanolamine (PE), allophycocyanin-peridin chlorophyll protein, PE-Cy5.5, PE-Cy7, or allophycocyanin-Cy7 (eBioscience, San Diego, CA). A FACScanto flow cytometer (BD Biosciences, Franklin Lakes, $\mathrm{NJ})$ was used to determine the cell populations, and the data were analyzed using FlowJo FACS Analysis software version 7.6.3 (Tree Star, Ashland, OR).

\section{ELISAs}

The $\alpha$-fodrin (JS-1), ${ }^{16}$ SS-A/Ro (Immunovision, Springdale, AR), SS-B/La (Immunovision), or single strand (ss)-DNA (Immunovision)-specific antibodies in sera from mice were measured using enzyme-linked immunosorbent assays (ELISAs), as previously described. ${ }^{16}$ In brief, the antibodies were added to microtiter wells at a concentration of $1 \mu \mathrm{g} / \mathrm{mL}$ of phosphate-buffered saline. Non-specific sites were absorbed with $1 \%$ bovine serum albumin in phosphate-buffered saline. After the plates were washed, diluted sera were added. Horseradish peroxidase-conjugated anti-mouse IgG (heavy and light chains; Vector, Burlingame, CA) was added as the 
secondary antibody, and o-phenylenediamine (Sigma-Aldrich) buffer was added. Antibodies were measured with a microplate reader (Bio-Rad Laboratories, Richmond, CA).

\section{Quantitative RT-PCR}

Total RNA was extracted from white adipose tissue (WAT), spleen, salivary glands, and liver using Isogen (Wako Pure Chemical Industries, Osaka, Japan) and subsequently reverse transcribed. The levels of mRNAs encoding F4/80, monocyte chemotactic protein (MCP)-1, Toll-like receptor (TLR)-4, NF- $\kappa$ B, IL-6, IL-1 $\beta$, interferon (IFN)- $\gamma$, tumor necrosis factor (TNF)- $\alpha$, IL-10, transforming growth factor (TGF)- $\beta$, and $\beta$-actin were determined using a PTC-200 DNA Engine Cycler (Bio-Rad Laboratories) with SYBR Premix Ex Taq (Takara Bio, Shiga, Japan). The primer sequences used were as follows: F4/80, 5'-CTTTGGCTATGGGCTTCCAGTC-3' (forward) and 5'-GCAAGGAGGACAGAGTTTATCGTG-3' (reverse); MCP-1, 5'-CTGGATCGGAACCAAATGAG- $3^{\prime}$ (forward) and $5^{\prime}$-TGAGGTGGTTGTGGAAAAGG-3' (reverse); TLR-4, 5'-CAGTGGTCAGTGTGATTGTGG-3' (forward) and $5^{\prime}$-TTCCTGGATGATGATGTTGGCAGC- $3^{\prime}$ (reverse); NF- $\kappa$ B, $5^{\prime}$-ATGGCAGACGATGATCCCTA- $3^{\prime}$ (forward) and 5'-TAGGCAAGGTCAGAATGCAC-3' (reverse); IL-1 $\beta, 5^{\prime}$-TGATGAGAATGACCTGTTCT-3' (forward) and 5' ${ }^{\prime}$-CTTCTTCAAAGATGAAGGAAA-3' (reverse); IL-6, 5'-GCTACCAAACTGGATATAATCAGGA-3' (forward) and $5^{\prime}$-CCAGGTAGCTATGGTACTCCAGAA-3' (reverse); IFN- $\gamma$, $5^{\prime}$-AGCGGCTGACTGAACTCAGATTGTAG-3' (forward) and $5^{\prime}$-GTCACAGTTTTCAGCTGTATAGGG-3' (reverse); IL-10, $5^{\prime}$-ATCGATTTCTCCCCTGTGAA- $3^{\prime}$ (forward) and $5^{\prime}$-TGTCAAATTCATTCATGGCCT- $3^{\prime}$ (reverse); TGF- $\beta$, $5^{\prime}$-GACCGCAACAACGCCATCTAT-3' (forward) and 5'GGCGTATCAGTGGGGGTCAG-3' (reverse); arginase-1, $5^{\prime}$-CAGAAGAATGGAAGAGTCAG-3' (forward) and 5'-CAGATATGCAGGGAGTCACC-3' (reverse); Ym-1, 5'CAGGTCTGGCAATTCTTCTGAA-3' (forward) and 5'-GTCTTGCTCATGTGTGTAAGTGA-3' (reverse); Fizz-1, 5'TCCCAGTGAATACTGATGAGA-3' (forward) and $5^{\prime}$-CCACTCTGGATCTCCCAAGA- $3^{\prime}$ (reverse); and $\beta$-actin, $5^{\prime}$ GTGGGCCGCTCTAGGCACCA-3' (forward) and 5'-CGGTTGGCCTTAGGGTTCAGGGGG-3' (reverse).

\section{Confocal Microscopy}

Frozen sections of adipose tissues were fixed using a cold acetone and methanol (1:1) mixture. Whole mounts of WAT were minced into small pieces, fixed in $4 \%$ paraformaldehyde, permeabilized with $1 \%$ Triton X-100 (Thermo Scientific, Waltham, MA) for 1 hour, and blocked with $5 \%$ goat serum. The frozen sections or whole adipose tissues were stained with Alexa 488-conjugated anti-mouse F4/80 antibodies (eBioscience). The nuclear DNA was stained with DAPI (Molecular Probe Inc., Eugene, OR). The tissues were counterstained for 1 hour with $5 \mu \mathrm{mol} / \mathrm{L}$
BODIPY 558/568 C12[4,4-difluoro-5-(2-thienyl)-4-bora-3a, 4a-diaza-s-indacene-3-dodecanoic acid] (Invitrogen, Carlsbad, CA) to visualize adipocytes. The images were acquired using an LSM 5 PASCAL confocal laser-scanning microscope (Carl Zeiss, Jena, Germany).

\section{Oil Red 0 Staining}

For staining of lipids, the frozen sections of salivary gland from WT and ArKO mice were fixed with $10 \%$ phosphatebuffered formaldehyde, incubated for 1 minute in $60 \% 2$ propanol, stained for 15 minutes with Oil Red O solution (Sigma-Aldrich), rinsed in 60\% 2-propanol, and washed with water. The specimens were counterstained with Mayer's hematoxylin solution for 5 minutes.

\section{IHC Analysis}

For immunohistochemical (IHC) analysis of MCP-1 in salivary gland, paraffin-embedded sections were deparaffinized and subsequently applied to heat-induced antigen retrieval in $10 \mathrm{mmol} / \mathrm{L}$ citrate buffer ( $\mathrm{pH}$ 6.0). The sections were incubated with rabbit MCP-1 polyclonal antibody (BioSS, Dundee, Scotland) or control rabbit immunoglobulin, subsequently developed using horseradish peroxidaseconjugated anti-rabbit immunoglobulin (Vector Laboratories Ltd, Peterborough, UK), and counterstained with methyl green.

\section{Statistical Analysis}

Statistical significance was determined using an unpaired Student's $t$-test.

\section{Results}

Inflammatory Lesions of Lacrimal and Salivary Glands in ArKO Mice

ArKO mice spontaneously develop SS-like lesions in their lacrimal and salivary glands. ${ }^{15}$ Herein, using another line of ArKO mice, ${ }^{17}$ lacrimal and salivary gland tissues were histopathologically analyzed until the mice reached 18 months of age. Severe inflammatory lesions with lymphocyte infiltration and tissue destruction in the lacrimal and salivary glands of $A r \mathrm{KO}$ mice were observed during the first 12 months; they resemble those of human SS (Figure 1A). Although slight or mild inflammation of the lacrimal and salivary glands was observed in WT mice older than 12 months, severe inflammatory lesions significantly increased in $\mathrm{ArKO}$ mice of the same age (Figure 1B). Many $\mathrm{CD}^{+} \mathrm{T}$ cells and $\mathrm{B} 220^{+}$cells infiltrated the salivary glands of the ArKO mice (Supplemental Figure S1A). Moreover, significantly higher levels of autoantibodies, such as anti-SSA, anti-SSB, and anti-ssDNA, were detected in the sera of ArKO mice compared with those in the sera of WT mice 
A

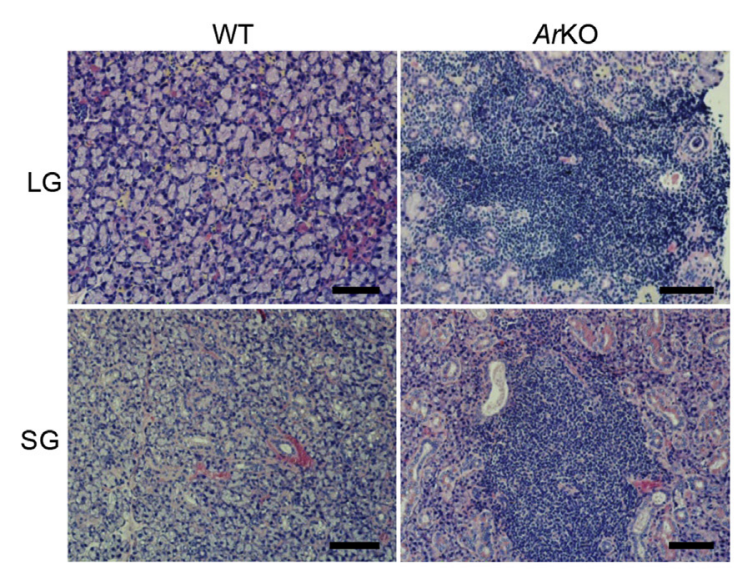

C

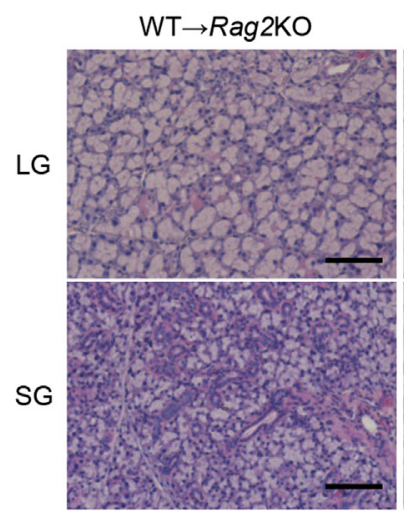

B
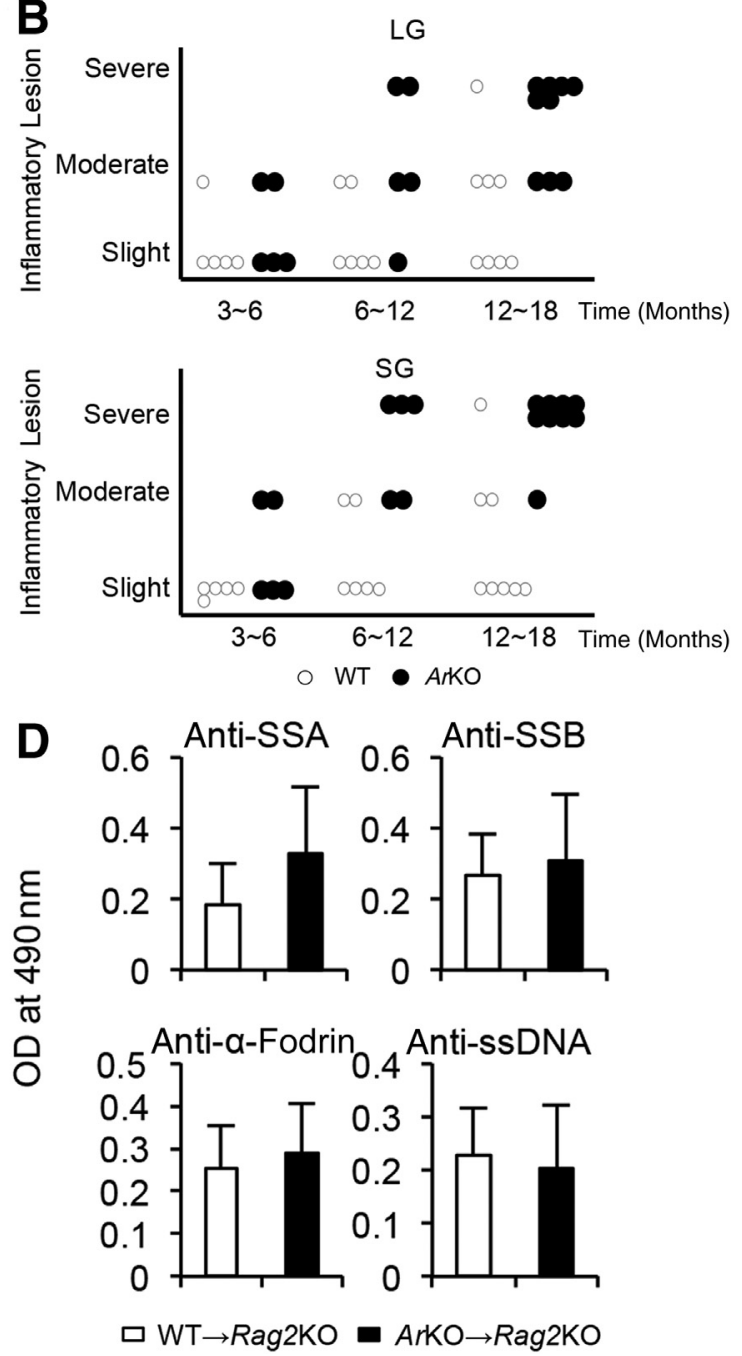

Figure 1 SS-like lesions in the lacrimal glands (LGs) and salivary glands (SGs) of ArKO mice. A: Severe inflammatory lesions in the LGs and SGs of ArKO mice. Shown are representative images of H\&E-stained sections of 12-month-old WT and ArKO mice. B: Pathological score of inflammatory lesions in LGs and SGs was evaluated using H\&E-stained sections of female WT and ArKO mice from 3 to 18 months of age (WT, $n=19 ;$ ArK0, $n=19)$. C: Spleen cells (5 $\left.\times 10^{6}\right)$ from WT and ArKO mice were i.v. transferred into female Rag2KO mice at 8 weeks of age. Pathological analysis of the LGs and SGs of the recipient Rag2KO mice was performed 3 months after transfer. Images are representative of each recipient group $(n=5)$. D: Autoantibody production (anti-SSA, anti-SSB, antissDNA, and anti- $\alpha$-fodrin antibodies) in the sera of recipient Rag2KO mice was analyzed using ELISAs. Data are shown as the means \pm SD of five mice of each group. Scale bar $=200 \mu \mathrm{m}(\mathbf{A})$.

(Supplemental Figure S1B). These findings are consistent with the results of another study that demonstrated that hyperactivation of B cells contributes to the onset of SS-like autoimmune lesions in lacrimal and salivary glands of ArKO mice. $^{15}$

To determine the contribution of peripheral immune cells, including $\mathrm{B}$ cells, to the onset of autoimmunity in ArKO mice, $5 \times 10^{6}$ spleen cells from WT or ArKO mice were i.v. transferred into Rag2KO mice. Twelve weeks after transfer, all organs were pathologically analyzed. Neither WT nor ArKO spleen cells induced inflammatory lesions in the lacrimal or salivary glands of the recipient mice (Figure 1C). Furthermore, there were no differences in the serum levels of autoantibodies, including anti-SSA, anti-SSB, anti- $\alpha$-fodrin, and anti-ssDNA (Figure 1D). ${ }^{9}$ Moreover, transfer of bone marrow cells from ArKO mice into irradiated $\operatorname{Rag} 2 \mathrm{KO}$ mice could not induce inflammatory lesions in salivary glands (Supplemental Figure S2A). In addition, the serum level of autoantibodies of the recipient mice transplanted with bone marrow cells from ArKO mice was similar to that from WT mice (Supplemental Figure S2B). These findings suggest that the transfer of immune cells from ArKO mice failed to induce SS-like lesions in the target exocrine glands. Moreover, they suggest that other factors influence the onset or development of inflammatory lesions in ArKO mice.

\section{Relationship between Adipose Tissue and Immune Cells of ArKO Mice}

Aromatase is abundantly present in the adipose tissues, ${ }^{18}$ and the adipocytes of $A r \mathrm{KO}$ mice are significantly larger and more 
abundant compared with those of WT mice. ${ }^{14}$ Furthermore, monocytes or cytokines in adipose tissue contribute to the pathogenesis of autoimmune disease. ${ }^{19,20}$ Therefore, we focused on adipose tissues to determine whether they were involved in inducing the SS-like lesions in ArKO mice. The volume of abdominal WAT in female ArKO mice at 12 months of age was greater than that of WT mice (Figure 2A). There was a slight, but insignificant, increase in body weight of $A r \mathrm{KO}$ mice compared with WT mice. However, the weight of WAT of ArKO mice was significantly increased (Figure 2B). Moreover, flow cytometric analysis showed that the proportion of dendritic cells (DCs) and macrophages in the WAT of ArKO mice was higher than that of WT mice (Figure 2C), as well as the absolute numbers of DCs and macrophages (Figure 2D). These findings suggest that increased numbers of DCs and macrophages in WAT may influence the onset or development of SS-like lesions.

\section{Expression of mRNAs Encoding Macrophage-Associated Proteins in WAT of ArKO Mice}

To understand the characteristics of macrophages that accumulated in the adipose tissues of ArKO mice, the expression levels of macrophage-associated genes were quantified using real-time RT-PCR. We detected significantly increased expression of mRNAs encoding cell surface glycoprotein F4/ 80 and MCP-1 in the WAT of ArKO mice compared with WT mice (Figure 3A). The expression levels of mRNAs encoding TLR-4 and NF- $\kappa \mathrm{B}$ in $\mathrm{ArKO}$ mice were significantly higher than in WT mice (Figure 3A).

Macrophage phenotypes are defined as M1 and M2, according to their production of certain cytokines. ${ }^{21}$ M1 macrophages produce the proinflammatory cytokines IL-1 $\beta$, IL-6, IFN- $\gamma$, and TNF- $\alpha$. In contrast, M2 macrophages produce the anti-inflammatory cytokines IL-10 and TGF- $\beta$. The expression levels of mRNAs encoding IL-1 $\beta$, IL- 6 , IFN- $\gamma$, and TNF- $\alpha$ mRNA in WAT of ArKO mice were significantly increased compared with those in WT mice (Figure 3B). In contrast, expression levels of mRNAs encoding IL-10 and TGF- $\beta$ mRNA of WAT in ArKO mice were significantly reduced (Figure $3 \mathrm{~B}$ ). In addition, there was no significant difference in mRNA expression of M2-macrophage markers, such as arginase-1, Fizz-1, and Ym-1, in WAT between WT and ArKO mice (Figure 3B). These findings suggest that aromatase regulates the migration and differentiation of macrophages in adipose tissues as well as their subsequent activation.

\section{Macrophages in Adipose Tissues of ArKO Mice}

The distribution of macrophages in WAT was evaluated using immunofluorescence microscopy. H\&E staining of adipose tissue sections revealed the accumulation of mononuclear cells around adipocytes in ArKO mice (Figure 4A). Moreover, the accumulated macrophages formed a crown-like structure in the WAT of ArKO mice; this was detected using DAPI (red) staining and an anti-F4/80 monoclonal antibody (green) (Figure 4A). Confocal microscopy revealed that accumulation of $\mathrm{F} 4 / 80^{+}$macrophages surrounding adipocytes stained with BODIPYR (blue) (Figure 4A). CCR2, a marker of M1 macrophages, was expressed at significantly higher levels by F4/ $80^{+}$cells in the WAT of ArKO mice compared with WT mice. In contrast, CD206, a marker of M2 macrophages, was expressed at significantly lower levels in the WAT of ArKO mice than in WT mice (Figure 4B). The results suggest that the increased abundance of WAT in ArKO mice enhanced the migration and maturation of activated M1 macrophages.

\section{Effect of an AI on WAT and Salivary Glands}

MCP-1, produced by adipocytes or macrophages, plays a key role in chemotaxis of monocytes into adipose tissues. ${ }^{22}$ However, it is unclear whether aromatase/estrogens regulate the expression of MCP-1 in WAT. To address this question, we i.p. administered 0, 50, and $200 \mu \mathrm{g}$ of AI into female C57BL/6 mice daily for 1 week. By using real-time RTPCR, we subsequently determined the expression levels of $M c p-1$ mRNA in various organs. The levels of the mRNA encoding MCP-1 in WAT increased as a function of AI dose (Figure 5). The expression of Mcp-1 mRNA in the salivary gland, a target organ of SS, was enhanced by AI treatment;
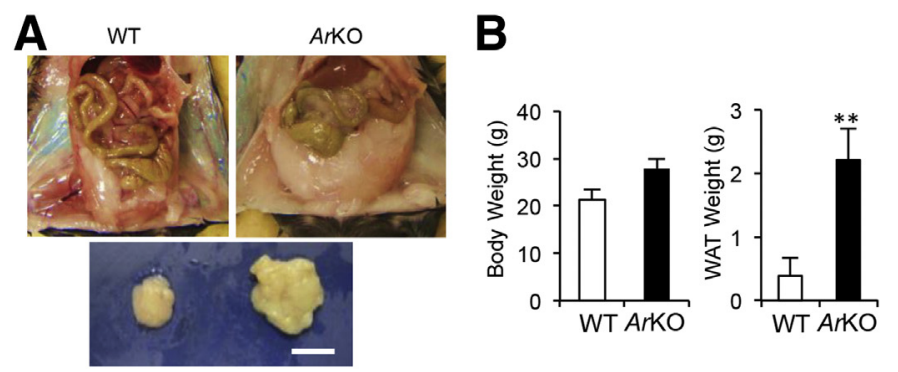

C

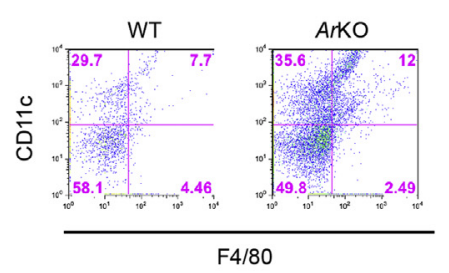

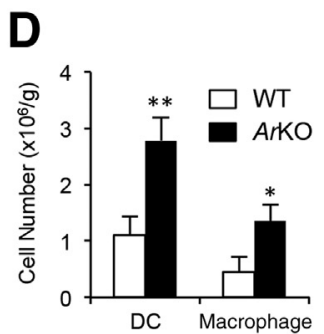

Figure 2 Adipose tissues of ArKO mice. A: Representative images of WAT of 12-month-old WT and ArKO mice (top panels). Bottom panel: Formalin-fixed tissues. B: Body weight and WAT weight of 12-month-old WT and ArKO mice. Data represent the means \pm SD of five mice from each group. C: The proportion of $\mathrm{CD} 11 \mathrm{C}^{+} \mathrm{F} 4 / 80^{-} \mathrm{DCS}$ or $\mathrm{F} 4 / 80^{+}$macrophages in WAT of WT and ArKO mice was analyzed using flow cytometry. Results are representative of five mice from each group. D: The number of DCs and macrophages in WAT of 12-month-old WT and ArKO mice. Cell number is shown as the means \pm SD of five mice of each group. ${ }^{*} P<0.05,{ }^{*} P<<0.005$. Scale bar $=1 \mathrm{~cm}(\mathbf{A})$. 

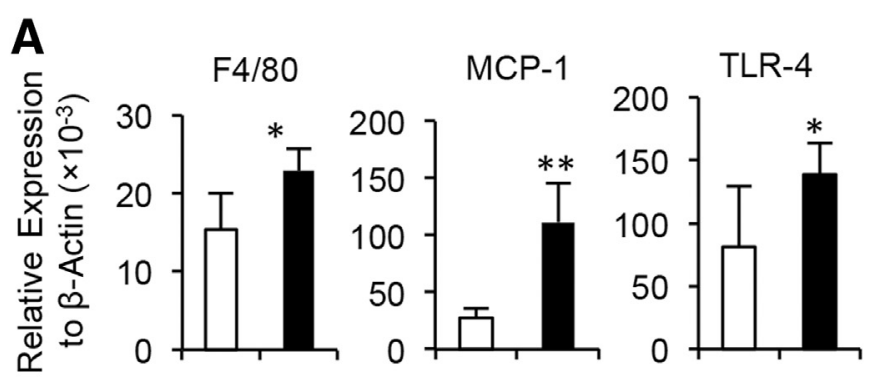

B

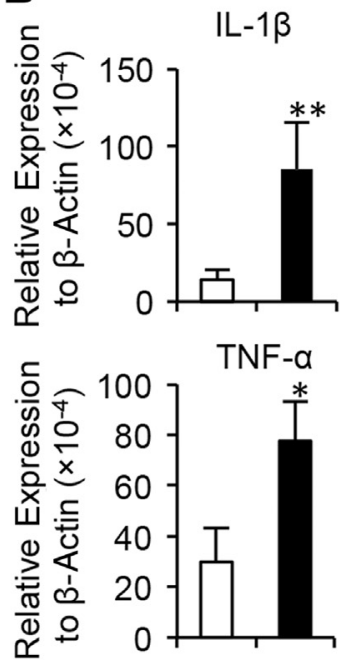

IL-6
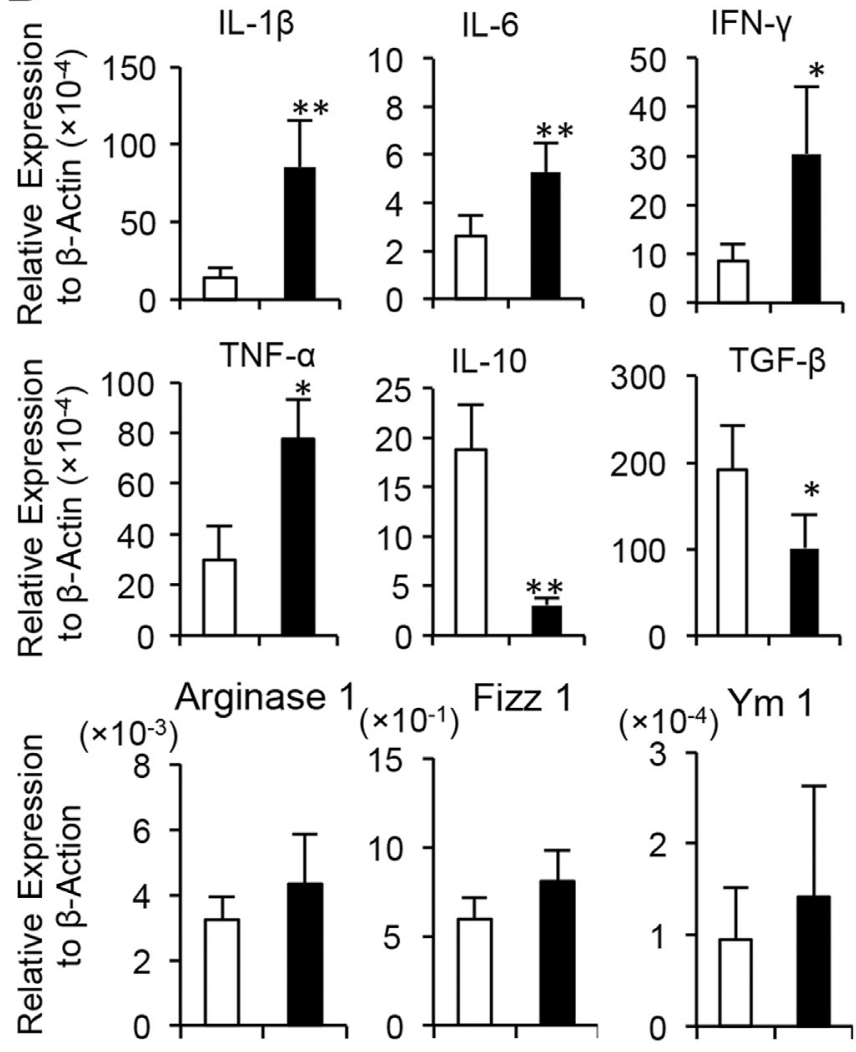

however, it was not enhanced in the liver and spleen (Figure 5). These findings demonstrate that the expression of MCP-1 in the salivary glands and in WAT is regulated by aromatase/estrogens.

\section{Expression of MCP-1 in Salivary Glands and Cervical WAT}

Abundant adipose tissue surrounds the salivary gland, and a change in fat levels is often observed in atrophic salivary glands because of chronic inflammation or aging. When the fatty change of the salivary gland in ArKO mice was compared with that in WT mice using Oil Red O staining, more salivary gland cells exhibited a fatty change in $A r K O$ mice (Figure 6A). Moreover, the expression level of $M c p-1$ mRNA of the salivary gland tissue of $A r \mathrm{KO}$ mice was significantly increased compared with that of WT mice (Figure 6B). A significantly increased number of F4/80 macrophages in the cervical WAT around the salivary

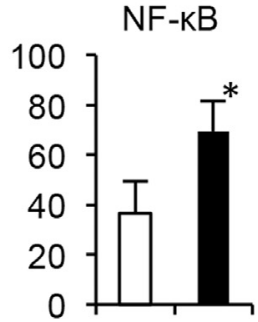

Figure 3 Real-time RT-PCR of the expression of monocyte-related genes in ArKO (black bars) and WT (white bars) mice. A: Levels of mRNAs encoding F4/80, MCP-1, TLR-4, and NF- KB in WAT of 12month-old WT and ArKO mice. B: The levels of mRNAs encoding IL- $1 \beta$, IL-6, IFN- $\gamma$, TNF- $\alpha$, IL-10, TGF- $\beta$, arginase- 1 , Fizz- 1 , and Ym- 1 in WAT of WT and $A r K 0$ mice. Data represent the means \pm SD of five mice from each group. ${ }^{*} P<0.05,{ }^{* *} P<0.005$. glands of $\mathrm{ArKO}$ mice was detected using immunofluorescence staining (Figure 6C). Furthermore, the expression of MCP-1 in the salivary gland of ArKO mice was significantly increased compared with that of WT mice (Figure 6D). In addition, macrophage markers of salivary gland tissues were analyzed by real-time RT-PCR. TLR- 4 mRNA of salivary gland from $\mathrm{ArKO}$ mice was significantly increased compared with that of WT mice (Supplemental Figure S3). There was no difference in mRNA expression, such as IFN- $\gamma$, TNF- $\alpha$, IL-10, and TGF- $\beta$, in the salivary gland tissues between WT and ArKO mice (Supplemental Figure S3). The findings suggest that MCP-1 may play a key role in the development of SS-like lesions in the salivary glands.

Effect of AI Administration on Autoimmune Lesions in Mouse Model of Primary SS

To confirm the role of aromatase in the SS-like lesions in the salivary and lacrimal glands, AI was administered into a 
A
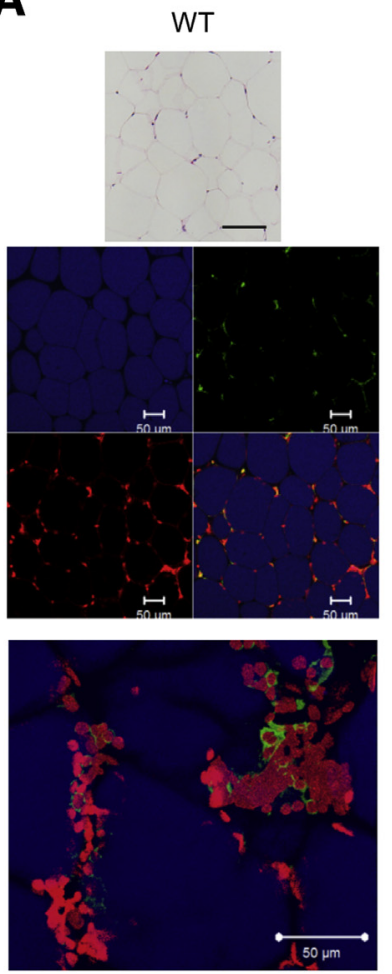

DAPI / F4/80 / Adipose tissue (BODIPYR)

WT

ArKO

B

Gated on $\mathrm{F} 4 / 80^{+}$

CCR2 (M1)
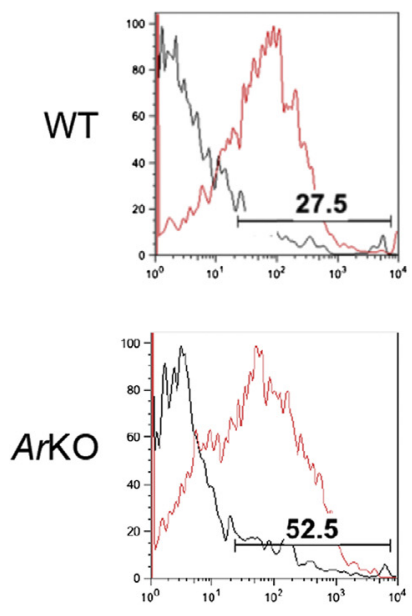

CCR2 (M1)
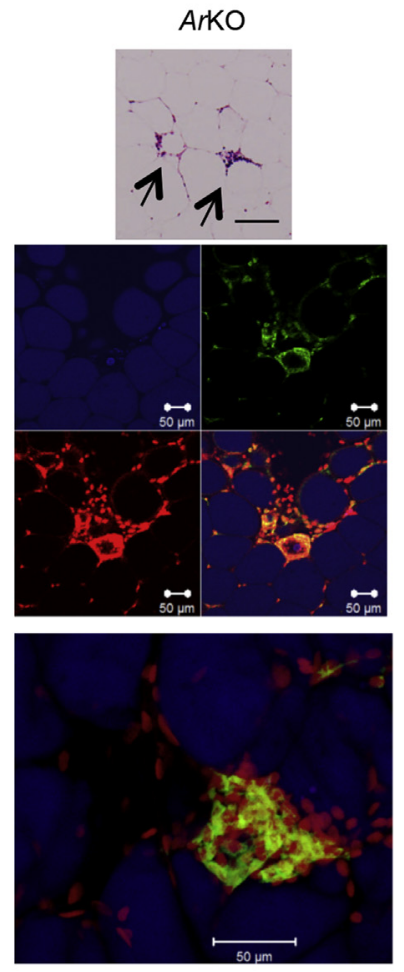

\section{)}


WAT

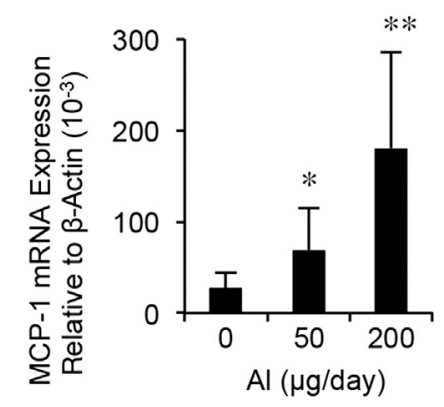

Liver

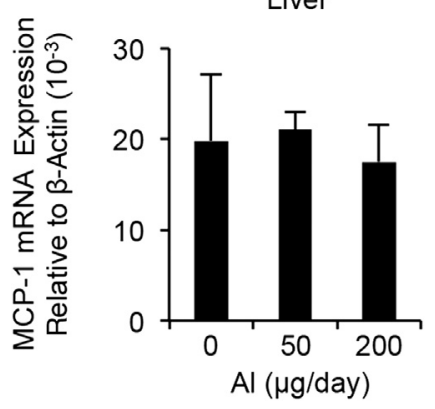

Salivary Gland

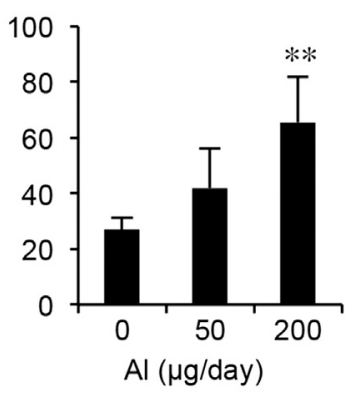

Spleen

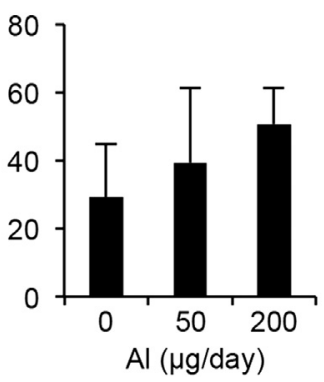

Figure 5 Effect of AI on the expression of MCP-1 mRNA in various tissues. Daily peritoneal injections of AI $(0,50$, and $200 \mu \mathrm{g}$ per mouse) were administered to 10-week-old female B6 mice for a week. The expression of MCP-1 mRNA in WAT, salivary glands, liver, and spleen was analyzed using real-time RT-PCR. Data represent the means \pm SD of five mice of each group. ${ }^{*} P<0.05,{ }^{* *} P<0.005$.

administration into ArKO mice results in preventing SS-like lesions, suggesting that estrogen may play a key role in the pathogenesis of autoimmune lesions of $A r K O$ mice. ${ }^{15} \mathrm{We}$ also observed SS-like inflammatory lesions in lacrimal and salivary glands and increased autoantibody production by $A r \mathrm{KO}$ mice. Moreover, adoptive transfer of both spleen cells and bone marrow cells from $A r \mathrm{KO}$ mice into Rag2KO mice failed to induce inflammatory lesions, suggesting that in $A r \mathrm{KO}$ mice factors other than immune cells contribute to the pathogenesis of SS-like disease. On the other hand, serum concentration of estradiol of $\mathrm{ArKO}$ mice at baseline is similar to that of WT mice in normal status. ${ }^{32}$ Although the estradiol or progesterone level of female WT mice is promoted in the estrus cycle, no change of the level is observed in female $\mathrm{ArKO}$ mice. ${ }^{32}$ It is believed that the hypothalamus-pituitary axis is disrupted in ArKO mice. Therefore, change of estrogen with diestrous/ estrous phase of the cycle is not seen in ArKO mice. In addition, it was reported that prolactin and testosterone levels of the sera in female ArKO mice are significantly elevated compared with those of WT mice. ${ }^{33}$ There may be any difference in the physiological function for immune response between estrogen and androgen. Previous reports demonstrated that administration of androgen protects autoimmune lesions in murine SS models. ${ }^{34,35}$ However, the function and metabolism of sex hormones are so complicated that the precise molecular mechanism of sex dimorphism in the onset of autoimmunity remains unclear.
It is well known that autoimmunity is a multifactorial disease. Although lack of estrogen is important for the onset or development of autoimmunity, the other factors influence the pathogenesis of the autoimmune disease. For instance, when neonatally thymectomized NFS/sld mice, one of the murine SS models, are ovariectomized during childbearing age, the autoimmune lesions are severely exaggerated. ${ }^{7}$ Herein, the incidence of autoimmune lesions in ArKO mice is largely increased after 6 months of age, suggesting that aging or an unknown factor may influence the onset or development of autoimmunity in the model. On the other hand, there is no report as for inflammatory lesions in estrogen receptor $\alpha$ or $\beta$ knockout. It is believed that autoimmune disease is triggered
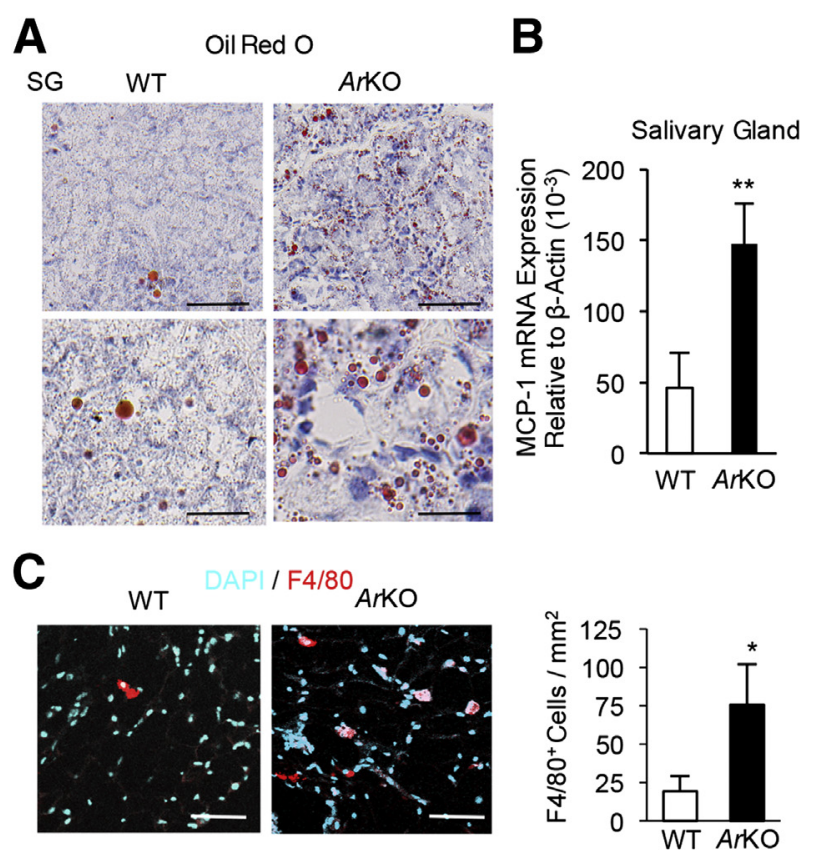

D

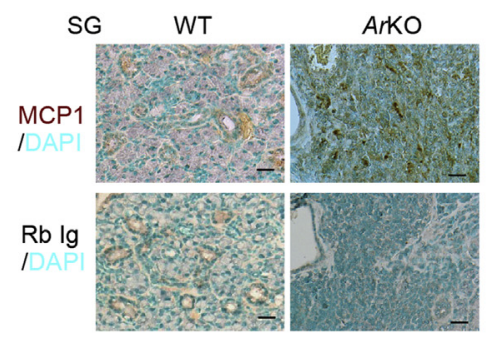

Figure 6 Relationship between fatty change and the expression of MCP1 in the salivary glands of $A r K O$ mice. A: Frozen sections of salivary glands in 10-month-old WT and ArKO mice were stained with Oil Red 0. Images represent three mice from each group. B: The expression of MCP-1 mRNA in salivary glands of WT and ArKO mice was analyzed using real-time RT-PCR. Data represent the means $\pm S D$ of three mice from each group. C: $F 4 / 80^{+}$ macrophages in the adipose tissue around salivary glands of WT and ArKO mice were detected using immunofluorescence staining. Nuclei were stained with DAPI. The number of $\mathrm{F} 4 / 80^{+}$cells $/ \mathrm{mm}^{2}$ was counted. Data represent the means \pm SD of five mice from each group. D: $M C P-1^{+}$cells in the salivary glands of WT and ArKO mice were detected using IHC analysis. Nuclei were stained with methyl green. ${ }^{*} P<0.05,{ }^{*} P<0.005$. Scale bars: $100 \mu \mathrm{m}(\mathbf{A}$, top panels); $20 \mu \mathrm{m}$ (A, bottom panels, and $\mathbf{D}) ; 50 \mu \mathrm{m}$ (C). Original magnifications: $\times 100$ (A, top panels); $\times 400$ (A, bottom panels). 

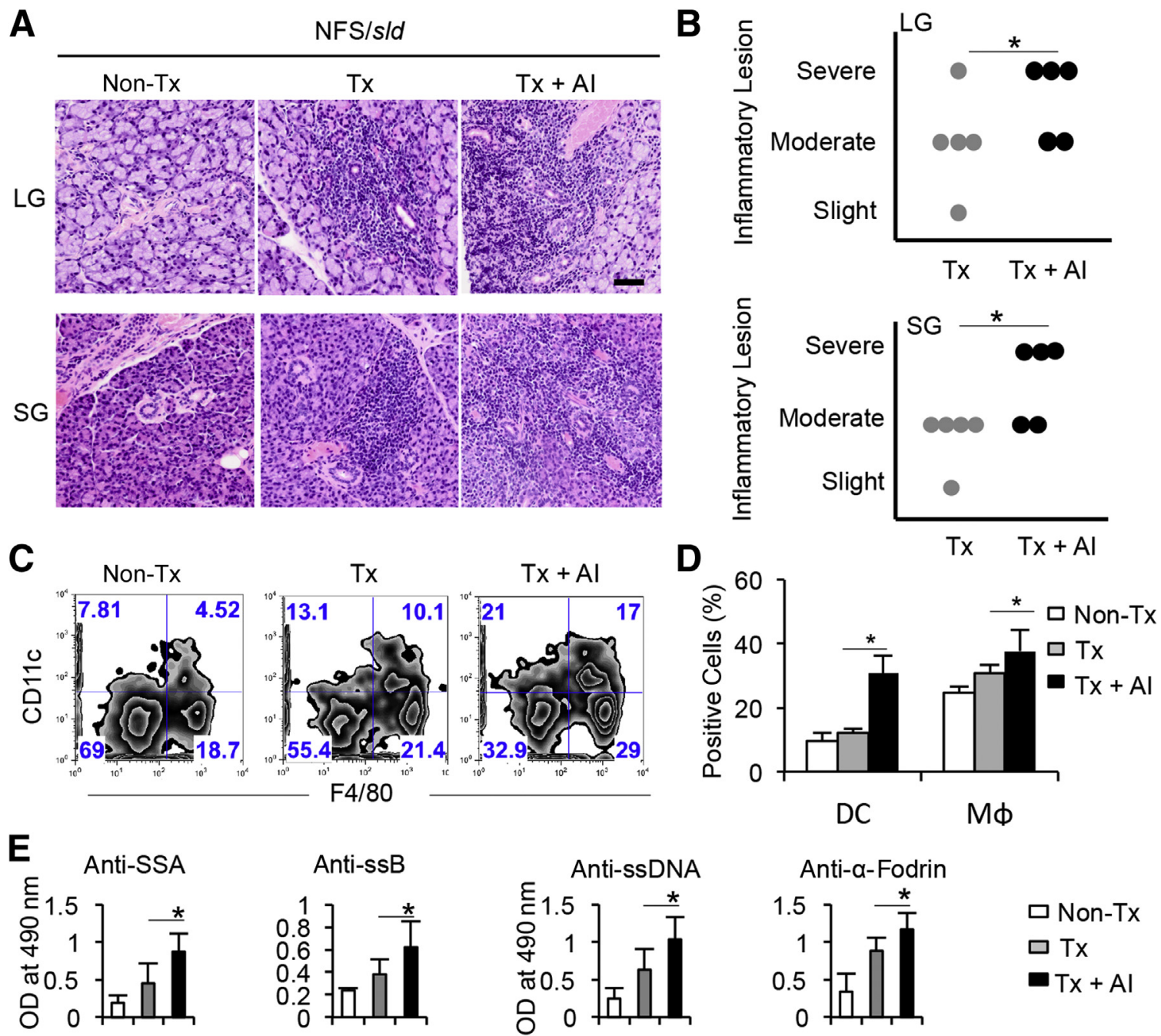

Figure 7 Effect of AI on the autoimmune lesions in SS-model mice. A: AI (200 $\mu \mathrm{g})$ dissolved in CMC was peritoneally injected daily into female Tx-NFS/sld mice 4 to 8 weeks of age. Control Tx-NFS/sld mice were injected with CMC. Pathological features of lacrimal glands (LGs) and salivary glands (SGs) were determined using H\&E staining. Images are representative of five mice from each group. B: Pathological grading was evaluated using H\&E-stained sections. Data represent the means \pm SD of five mice from each group. C: The proportion of monocytes that expressed CD11c and F4/80 was analyzed using flow cytometry. Results are representative of five mice from each group. $\mathrm{D}$ : $\mathrm{CD} 11 \mathrm{C}^{+} \mathrm{DCs}$ and $\mathrm{F} 4 / 80^{+}$macrophages were quantified using flow cytometric analysis. Data represent the means \pm SD of five mice from each group. E: Serum levels of anti-SSA, anti-SSB, anti- $\alpha$-fodrin, and anti-ssDNA antibodies were determined using ELISAs. Data represent the means $\pm \mathrm{SD}$ of five mice from each group. ${ }^{*} P<0.05$. Scale bar $=100 \mu \mathrm{m}(\mathbf{A})$. Original magnification, $\times 200(\mathbf{A})$.

by multiple factors. Although deficiency in estrogen/estrogen receptor signaling influences immune responses, only deficiency in the signaling cannot break the immune tolerance to induce autoimmunity. Aromatase may be related with various factors for the pathogenesis of SS-like lesions. However, further research on the basis of sex steroid hormone will be required for understanding the molecular pathogenesis of autoimmunity.

Aromatase is considerably expressed in adipose tissues, and ArKO mice develop marked abdominal adiposity, which is accompanied by increases in the weight of the gonadal and infrarenal fat pads. ${ }^{13,14}$ Adipose tissues in obesity disrupt the immune system and influence the pathogenesis of autoimmune disease. ${ }^{36,37}$ Therefore, we determined whether the presence of immune cells in adipose tissues plays a role in autoimmunity in ArKO mice. More important, similar to its effects on adipose tissue, we show that aromatase/estrogens control adiposity and the production of MCP-1 in the salivary gland, a target organ of SS. We speculate that salivary gland cells with adiposity controlled by aromatase may produce MCP-1 to attract macrophages to the target organ. Accumulated M1 macrophages might secrete inflammatory cytokines to enhance SS-like lesions and function as antigen-presenting cells in the target organ to develop SS-like lesions. These findings suggest that the specificity of the salivary gland as a target organ in SS may be explained by the estrogen deficiency-induced phenotypic change of salivary glands.

Excess adiposity increases the risk of developing metabolic syndrome, ${ }^{38}$ and obesity is a state of chronic systemic inflammation. Macrophages are considered a crucial component of adipose tissues involved in physiological and pathological remodeling. ${ }^{35}$ Monocytes are recruited to 
adipose tissues in obesity to become M1 macrophages and produce MCP-1, IL- $1 \beta$, IL- 6 , IFN- $\gamma$, and TNF- $\alpha$ to promote systemic proinflammatory signaling. ${ }^{39,40}$ In contrast, M2 macrophages produce IL-10 and TGF- $\beta$ to suppress inflammation. ${ }^{39,40}$ Because IL- $1 \beta$ and TGF- $\beta$ are enzymatically cleaved, the production of the tissues was measured by ELISA. Although IL-1 $\beta$ of WAT from ArKO mice was significantly increased compared with that from WT mice (WT versus $A r \mathrm{KO}, 120.3 \pm 39.2$ versus $191.3 \pm 52.3 \mathrm{pg} /$ WAT; $P<0.05)$, TGF- $\beta$ was undetectable. It seems to be difficult to quantify the concentration of TGF- $\beta$ in WAT. M1 macrophages contribute to increased insulin resistance in obesity, and M2 macrophages enhance insulin sensitivity through a peroxisome proliferator-activated receptor $\gamma$-dependent mechanism. ${ }^{22}$ The development of obesity in ArKO mice occurs at 3 months of age and is accompanied by a marked increase in the weights of WAT, suggesting that estrogen synthesis regulates lipid metabolism. ${ }^{14}$ However, the report did not indicate whether the increased weight of WAT influences the immune system. Herein, we report that the number of M1 macrophages in the WAT of ArKO mice increased compared with WT mice in addition to the increased WAT. Moreover, our findings suggest that the synthesis of MCP-1 by WAT plays a key role in attracting M1 macrophages to WAT, which induces the preferential production of proinflammatory cytokines.

The role of the aromatase-estrogen axis in the onset or development of autoimmune disease is poorly understood. DCs and macrophages, which are present in WAT and salivary glands, may play a key role in disrupting peripheral immune tolerance and triggering the onset or enhancing the development of SS-like lesions. Increased adiposity in the salivary gland, resembling that of the WAT in ArKO mice, suggests that changes occur in the salivary glands in postmenopausal women with SS. Furthermore, fatty change is observed in the salivary glands of women with SS. ${ }^{41}$ Furthermore, a clinical study observed that the expression of MCP-1 is significantly up-regulated in salivary gland tissues from patients with SS compared with those from healthy controls. ${ }^{42}$ Our findings suggest that in $A r K O$ mice, adiposity in the salivary gland was due to estrogen deficiency and further suggest the chain of events as follows: MCP-1 produced by the salivary gland and fat tissue attracted macrophages. The production of several proinflammatory cytokines by these macrophages disrupted local immune tolerance in the salivary gland to trigger the onset of SS. Therefore, estrogen deficiency caused by menopause may link the pathogenesis of SS to an imbalance of cytokine production by macrophages in adipose and target tissues. In addition, we previously demonstrated that estrogen deficiency triggers or promotes apoptosis of salivary gland cells. $^{8}$ Our preliminary experiment showed that increased apoptotic cells were found in aged ArKO mice compared with WT mice. Therefore, it is possible that a part of salivary gland cells with adiposity would undergo apoptosis. Aromatase deficiency results in the low estrogen level or estrogen deficiency. Physiological change of estrogen in females is not observed in ArKO mice. We think that the normal change of estrogen in females through aromatase maintains physiological homeostasis in immune system and fat metabolism. As described, aging is also related with the pathogenesis of autoimmunity in ArKO mice. Although the precise molecular mechanism is not clear, aromatase may play a key role in the onset or development of SS-like lesions through disturbance of immune cells, adipose tissue, and target organs.

In conclusion, aromatase deficiency resulted in the onset and development of SS-like autoimmune lesions through a switch to cytokine production by M1 macrophages in systemic adipose tissues and target organs. Our findings suggest that decrease of estrogens induced by menopause plays a key role in the pathogenesis of autoimmunity.

\section{Acknowledgments}

We thank Ai Katayama, Satoko Katada, and Michiko Kino for technical assistance.

\section{Supplemental Data}

Supplemental material for this article can be found at http://dx.doi.org/10.1016/j.ajpath.2014.09.006.

\section{References}

1. Waldmann H: Tolerance: an overview and perspectives. Nat Rey Nephrol 2010, 6:569-576

2. von Boehmer H, Melchers F: Checkpoints in lymphocyte development and autoimmune disease. Nat Immunol 2010, 11:14-20

3. Gleicher N: Postpartum depression, an autoimmune disease? Autoimmun Rev 2007, 6:572-576

4. Cutolo M, Sulli A, Straub RH: Estrogen metabolism and autoimmunity. Autoimmun Rev 2012, 11:A460-A464

5. Konttinen YT, Fuellen G, Bing Y, Porola P, Stegaev V, Trokovic N, Falk SS, Liu Y, Szodoray P, Takakubo Y: Sex steroids in Sjögren's syndrome. J Autoimmun 2012, 39:49-56

6. Fox RI: Sjögren's syndrome. Lancet 2005, 366:321-331

7. Ishimaru N, Saegusa K, Yanagi K, Haneji N, Saito I, Hayashi Y: Estrogen deficiency accelerates autoimmune exocrinopathy in murine Sjogren's syndrome through fas-mediated apoptosis. Am J Pathol 1999, 155:173-181

8. Ishimaru N, Arakaki R, Omotehara F, Yamada K, Mishima K, Saito I, Hayashi Y: Novel role for RbAp48 in tissue-specific, estrogen deficiency-dependent apoptosis in the exocrine glands. Mol Cell Biol 2006, 26:2924-2935

9. Ishimaru N, Arakaki R, Yoshida S, Yamada A, Noji S, Hayashi Y: Expression of the retinoblastoma protein RbAp48 in exocrine glands leads to Sjögren's syndrome-like autoimmune exocrinopathy. J Exp Med 2008, 205:2915-2927

10. Hill RA, Boon WC: Estrogens, brain, and behavior: lessons from knockout mouse models. Semin Reprod Med 2009, 27:218-228

11. Hill RA, Chua HK, Jones ME, Simpson ER, Boon WC: Estrogen deficiency results in apoptosis in the frontal cortex of adult female aromatase knockout mice. Mol Cell Neurosci 2009, 41:1-7

12. Bakker J, Pierman S, Gonzalez-Martinez D: Effects of aromatase mutation (ArKO) on the sexual differentiation of kisspeptin neuronal numbers and their activation by same versus opposite sex urinary pheromones. Horm Behav 2010, 57:390-395 
13. Jones ME, Thorburn AW, Britt KL, Hewitt KN, Wreford NG, Proietto J, Oz OK, Leury BJ, Robertson KM, Yao S, Simpson ER: Aromatase-deficient (ArKO) mice have a phenotype of increased adiposity. Proc Natl Acad Sci U S A 2000, 97:12735-12740

14. Misso ML, Murata Y, Boon WC, Jones ME, Britt KL, Simpson ER: Cellular and molecular characterization of the adipose phenotype of the aromatase-deficient mouse. Endocrinology 2003, 144:1474-1480

15. Shim GJ, Warner M, Kim HJ, Andersson S, Liu L, Ekman J, Imamov O, Jones ME, Simpson ER, Gustafsson JA: Aromatase-deficient mice spontaneously develop a lymphoproliferative autoimmune disease resembling Sjögren's syndrome. Proc Natl Acad Sci U S A 2004, 101:12628-12633

16. Kohashi M, Ishimaru N, Arakaki R, Hayashi Y: Effective treatment with oral administration of rebamipide in a mouse model of Sjögren's syndrome. Arthritis Rheum 2008, 58:389-400

17. Honda S, Harada N, Ito S, Takagi Y, Maeda S: Disruption of sexual behavior in male aromatase-deficient mice lacking exons 1 and 2 of the cyp19 gene. Biochem Biophys Res Commun 1998, 252: $445-449$

18. Bulun SE, Chen D, Moy I, Brooks DC, Zhao H: Aromatase, breast cancer and obesity: a complex interaction. Trends Endocrinol Metab 2012, 23:83-89

19. Toussirot E, Binda D, Gueugnon C, Dumoulin G: Adiponectin in autoimmune diseases. Curr Med Chem 2012, 19:5474-5480

20. Procaccini C, Carbone F, Galgani M, La Rocca C, De Rosa V, Cassano S, Matarese G: Obesity and susceptibility to autoimmune diseases. Expert Rev Clin Immunol 2011, 7:287-294

21. Morris DL, Singer K, Lumeng CN: Adipose tissue macrophages: phenotypic plasticity and diversity in lean and obese states. Curr Opin Clin Nutr Metab Care 2011, 14:341-346

22. Ota T: Chemokine systems link obesity to insulin resistance. Diabetes Metab J 2013, 37:165-172

23. Haneji N, Hamano H, Yanagi K, Hayashi Y: A new animal model for primary Sjögren's syndrome in NFS/sld mutant mice. J Immunol 1994, 153:2769-2777

24. Haneji N, Nakamura T, Takio K, Yanagi K, Higashiyama H, Saito I, Noji S, Sugino H, Hayashi Y: Identification of alpha-fodrin as a candidate autoantigen in primary Sjögren's syndrome. Science 1997, 276:604-607

25. Voulgarelis M, Tzioufas AG: Pathogenetic mechanisms in the initiation and perpetuation of Sjögren's syndrome. Nat Rev Rheumatol 2010, 6:529-537

26. Cutolo M, Sulli A, Capellino S, Villaggio B, Montagna P, Seriolo B, Straub RH: Sex hormones influence on the immune system: basic and clinical aspects in autoimmunity. Lupus 2004, 13:635-638
27. Nussinovitch U, Shoenfeld Y: The role of gender and organ specific autoimmunity. Autoimmun Rev 2012, 11:A377-A385

28. McCarthy M: The "gender gap" in autoimmune disease. Lancet 2000, 356:1088

29. Beagley KW, Gockel CM: Regulation of innate and adaptive immunity by the female sex hormones oestradiol and progesterone. FEMS Immunol Med Microbiol 2003, 38:13-22

30. Nadkarni S, McArthur S: Oestrogen and immunomodulation: new mechanisms that impact on peripheral and central immunity. Curr Opin Pharmacol 2013, 13:576-581

31. Stocco C: Tissue physiology and pathology of aromatase. Steroids 2012, 77:27-35

32. Fisher CR, Graves KH, Parlow AF, Simpson ER: Characterization of mice deficient in aromatase (ArKO) because of targeted disruption of the cyp19 gene. Proc Natl Acad Sci U S A 1998, 95:6965-6970

33. Mcpherson SJ, Wang H, Jones ME, Pedersen J, Iismaa TP, Wreford N, Simpson ER: Elevated androgen and prolactin in aromatase-deficient mice cause enlargement, but not malignancy, of the prostate gland. Endocrinology 2001, 142:2458-2467

34. Azzarolo AM, Wood RL, Mircheff AK, Richters A, Olsen E, Berkowitz M, Bachmann M, Huang ZM, Zolfagari R, Warren DW: Androgen influence on lacrimal gland apoptosis, necrosis, and lymphocyte infiltration. Invest Opthalmol Vis Sci 1999, 40:592-602

35. Mostafa S, Seamon V, Azzarolo AM: Influence of sex hormones and genetic predisposition in Sjögren's syndrome: a new clue to the immunopathogenesis of dry eye disease. Exp Eye Res 2012, 96:88-97

36. Derdemezis CS, Voulgari PV, Drosos AA, Kiortsis DN: Obesity, adipose tissue and rheumatoid arthritis: coincidence or more complex relationship? Clin Exp Rheumatol 2011, 29:712-727

37. Stofkova A: Leptin and adiponectin: from energy and metabolic dysbalance to inflammation and autoimmunity. Endocr Regul 2009, 43:157-168

38. Bartelt A, Heeren J: Adipose tissue browning and metabolic health. Nat Rev Endocrinol 2013, 10:24-36

39. Chawla A, Nguyen KD, Goh YP: Macrophage-mediated inflammation in metabolic disease. Nat Rev Immunol 2011, 11:738-749

40. Han JM, Levings MK: Immune regulation in obesity-associated adipose inflammation. J Immunol 2013, 191:527-532

41. Izumi M, Eguchi K, Nakamura H, Nagataki S, Nakamura T: Premature fat deposition in the salivary glands associated with Sjögren syndrome: MR and CT evidence. AJNR Am J Neuroradiol 1997, 18:951-958

42. Iwamoto N, Kawakami A, Arima K, Nakamura H, Kawashiri SY, Tamai M, Kita J, Okada A, Koga T, Kamachi M, Yamasaki S, Ichinose K, Ida H, Origuchi T, Eguchi K: Regulation of disease susceptibility and mononuclear cell infiltration into the labial salivary glands of Sjögren's syndrome by monocyte chemotactic protein-1. Rheumatology (Oxford) 2010, 49:1472-1478 